\title{
Studies regarding the processing of small thickness containers
}

\author{
Mihaela Urdea $^{1, *}$ \\ ${ }^{1}$ Transilvania University of Braşov, Department of Manufacturing Engineering, Mihai Viteazul No.5, \\ Braşov, Romania
}

\begin{abstract}
This paper studies the possibility of modeling containers made of small thickness sheet metal for the final goal to process them. It's about large external buffer containers for products that come or leave by truck. Modeling is done in Sheet Metal module and it is based on the possibility of obtaining the containers' unfolded components. It studies the issue of the efficient use of sheet metal with standard dimensions in the context of an optimal processing. These containers, completely built of sheet metal, are fabricated in a variety of sizes and are made up of welded parts. In this paper different varieties of components are defined in order to develop a design strategy for a rapid modeling which will result in a rapid construction. This study was conducted with the participation of Raptronic Process Engineering Company.
\end{abstract}

\section{The use and appearance of containers}

Boxes and containers for transport and storage goods, for example the containers manufactured at Raptronic Process Engineering Company, can be modeled in SolidWorks, using the Sheet Metal tools. Figure 1 presents a general type for these containers [1].

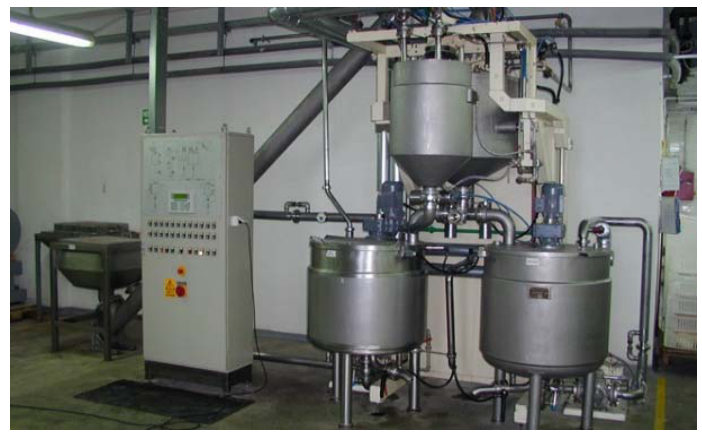

Fig. 1. Containers for goods.

Raptronic Process Engineering is specialized in the development and manufacturing of equipment and process technologies for food, chemical and pharmaceutical industries. RPE

* Corresponding author : urdeam@unitbv.ro 
produces customized containers or tanks, bunkers, agitators, kneading and blending machines of different capacities, made out of stainless steel and manufactured based on the customers' needs. RPE delivers automatic carbonation and pre-mix equipment for any types of liquid products, functioning at certain pressures or adjustable pressures. In the transport activity large containers are used [1].

These containers are completely built of sheet metal and can be fabricated in a variety of sizes and are made up of welded parts. It is adopted the general shape of the containers with weldable components according to Figure 2 in which 4 major sub-assemblies can be notice: the cover with the locking system, the body, the base and 3 legs. There are observed as useful components a spigot outlet, 2 sensor levels, an elbow for ventilation, a manhole, etc.
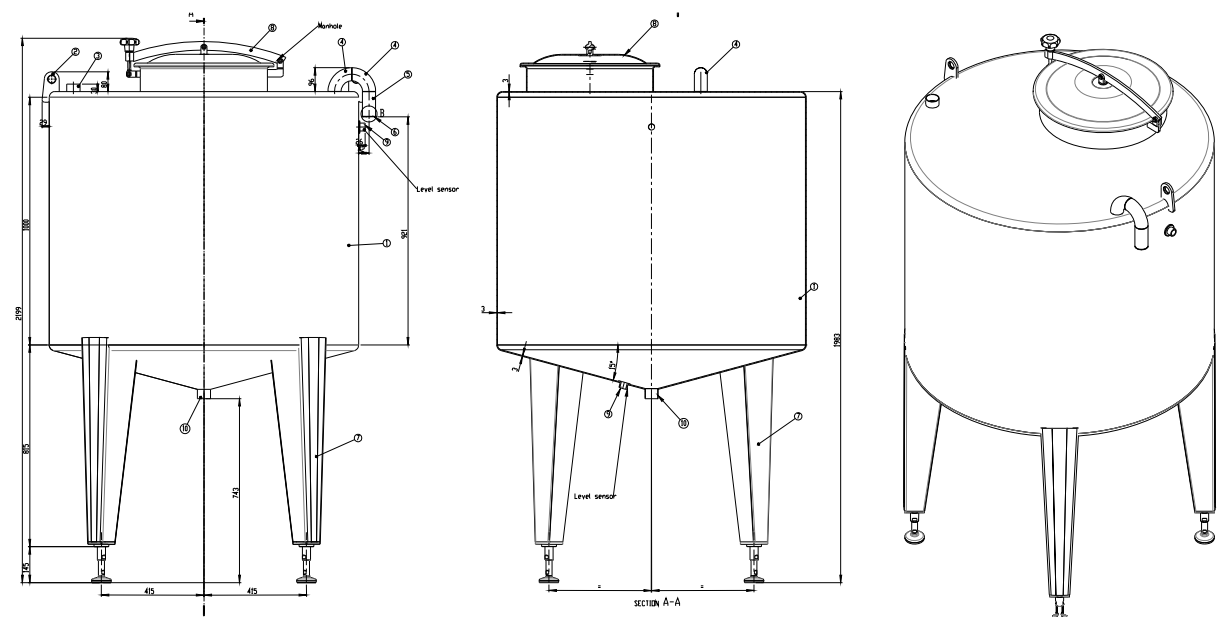

Fig. 2. Container represented in AutoCAD.

The sheet metal bodies can be easily processed and are also easy to be modeled in SolidWorks. Sheet Metal tools contain specialized functions for modeling sheet metal parts. The modeling process starts from a basic pattern, similar to the conventional solid modeling, which is developed through the addition of new entities. The studied containers are large exterior buffer containers for products that are transported by truck and have different shapes and dimensions.

The constant thickness of the walls allows a special strategy for the modeling selection and similarly a design strategy depending on the blank material available on the market. Also the finished product must be studied and processed from the point of view of internal cleaning systems (CIP S).

\section{The container}

In the sheets metal modeling activity, Sheet Metal tools can be used. There are some exceptions depending on the components shape. In this case the sheet parts can be modeled from a solid wall thickness, without using the specific advanced functions of Sheet Metal tools. Finally SolidWorks offers the possibility of transforming the parts in Sheet Metal tools. The alloy steel, symbolized AISI 304 in SolidWorks, is the used material for the studied containers.

Studying in this context the welded components of the containers, it was noticed that the transformation in Sheet Metal tools is not always possible. This paper offers some steps in 
this transformation, and the study is made permanently from the viewpoint of the blank material.

As a sheet blank it can be used sheet with the following dimensions: 1000x2000, $1250 \times 2500,1500 \times 3000$, but often it is preferable role sheet metal, due to uncommon sizes $[1]$.

In case of the fluid container, its body can be built from more overlapping welded cylinders, with different thicknesses for every segment, increasing towards the base. This paper focuses on designing the body components with constant thickness.

\subsection{The cylinder}

In the situation of constant thickness, the cylindrical part of the container can be modeled easily, like a sheet model, in two ways; using only the Sheet Metal tools (Fig. 3 a) or using first the Part tools and after it the Sheet Metal tools (Fig. 3 b).
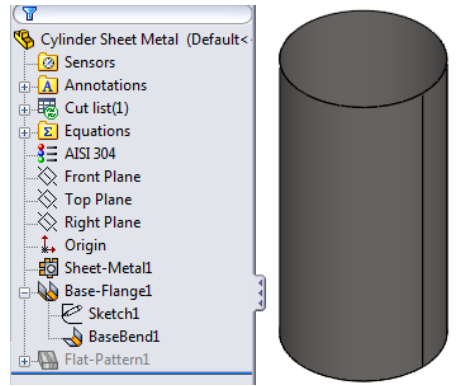

a
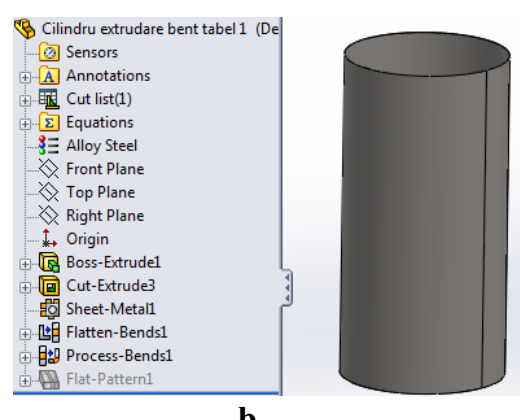

b

Fig. 3. The cylindrical part of the container.

The final goal is to obtain the flattened cylinder, cut on the height and defining the developed dimensions in order to choose the blank. With Flatten function one can individually flatten any component in a multibody sheet metal part (Fig. 4). Another purpose is to measure the blank dimensions, necessary for obtaining this component.
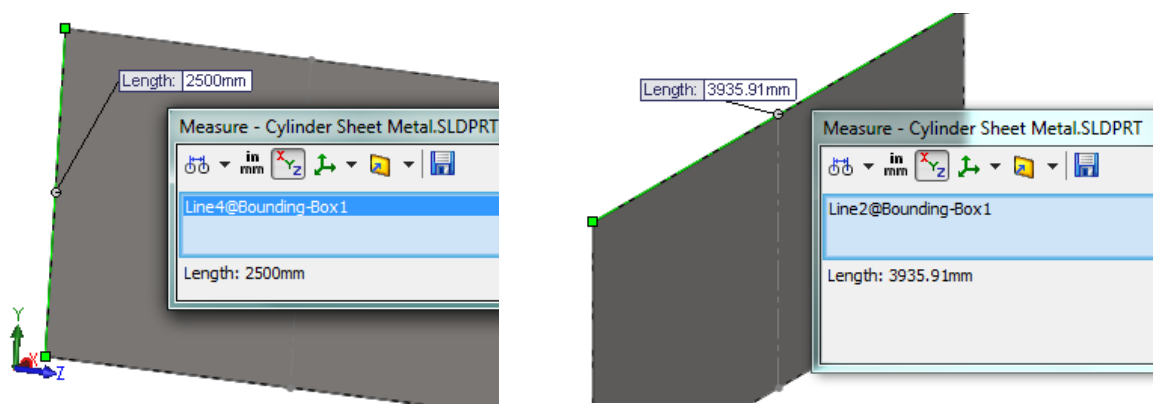

Fig. 4. The flat pattern for the cylinder, measured with Measure function.

For the body both versions are preferred considering several modeling options for a cylindrical shape using the Design Table tools. Due to the fact that the designer uses several sizes of the containers a table of sizes will be generated. For this purpose, all sizes are defined as variable using Link-value. The Design Table tools will develop an Excel table with the linked values (Fig. 5 a). Also considering the possible width of the sheet blank it will assign a relationship related on the cylinder height. 


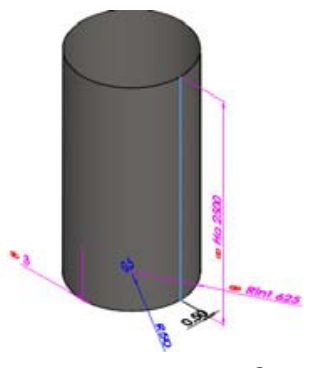

$\mathbf{a}$

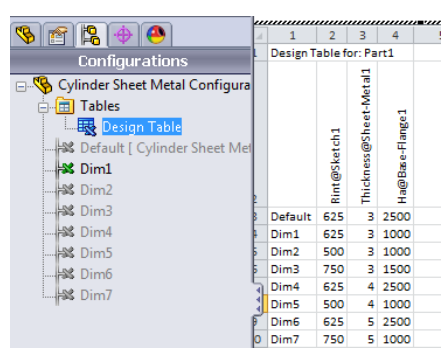

1000

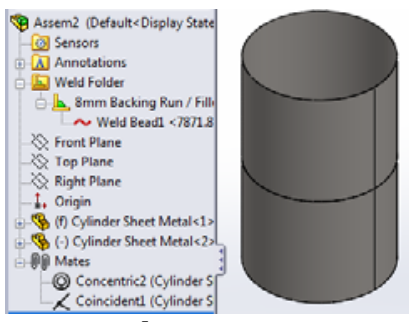

b

Fig. 5. Design Table for the cylinder.

In the same way all variable components of the container will be processed. So, these flattened parts will be defined and measured. For cylinders with great heights, without sheet blank dimensions, welded sheet metal is used (Fig. 5 b).

\subsection{The container bottom}

The container bottom is a tapered surface, rounded at the edges for joining by welding with the cylinder. It can be noticed that the only way for obtaining the base with the proposed shape, is the classic Part way. The Sheet Metal functions cannot process a profile like this, but in this situation the flattened shape will not be obtained. To solve this problem a perfect conical shape which can be modeled by Sheet Metal, for the base, was proposed. It will then be performed a bending for joining by welding with cylinder.

Figure 6 presents the base sketch with the calculated dimensions before the bending for the variant Dim 625. In the graphic construction the sketch length is $633.8 \mathrm{~mm}$.

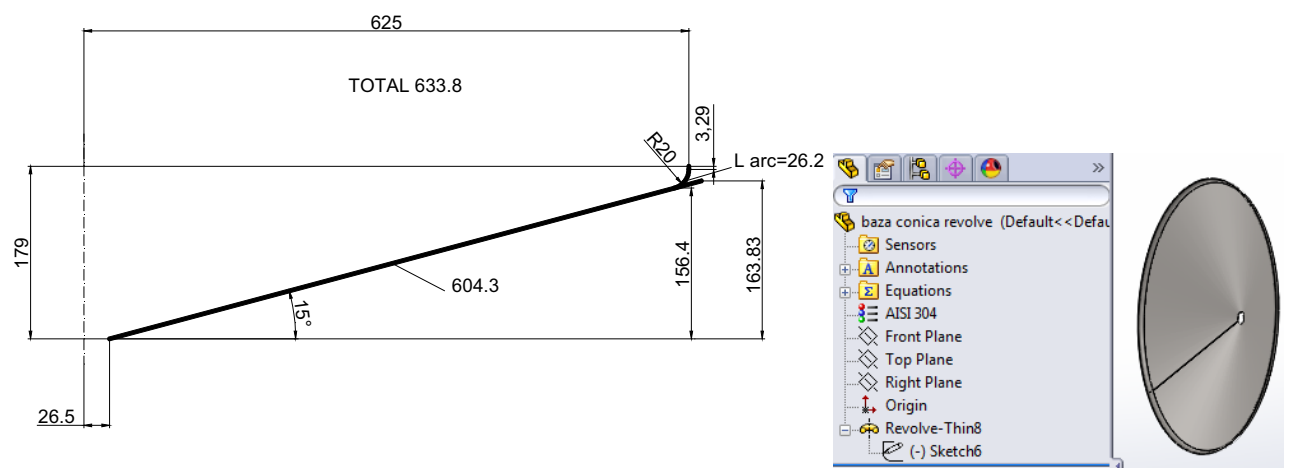

Fig. 6. The container bottom before and after bending.

$\mathrm{K}-$ Factor is a ratio that represents the location of the neutral sheet with respect to the thickness of the sheet metal part. In case of using sheet metal bending K-Factor, the following calculations result (Fig. 7) [2, 3]:

$$
\begin{gathered}
\text { Bend Allowance }=\pi(R+K T) * A / 180=\pi(20+1.5) * 75 / 180=4.581 \\
\text { Total length }=604.3+3.29+\text { Bend Allowance }=612.171
\end{gathered}
$$

It can be noticed a difference between the two studied situations, due to stretching of the material during bending. 


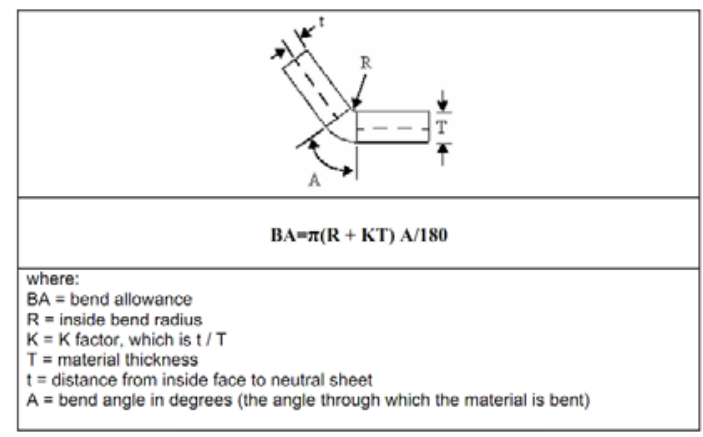

Fig. 7. Information about the K-Factor.

It is measured the dimensions of the cone before and after flattening. Measure function makes all the necessary calculations for the cone and blank (Fig. 8).
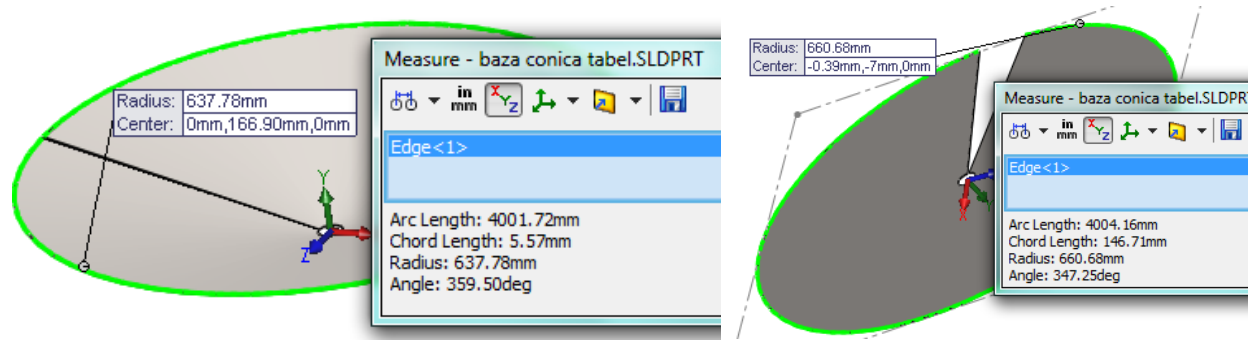

Fig. 8. Measure function used for measuring the size of the cone and of the blank.

The shown dimensions are for the studied shape, the inner diameter for cylinder 1250 $\mathrm{mm}$. Also in this case it has been developed a table of dimensions related to the cylinder table with 7 dimensions.

Similarly, the cover was modeled and measured.

\subsection{The container foot}

Figure $9 \mathrm{a}$, is an example of a sheet metal foot used for small capacity containers. Also, to this component a Design Table, according to the possible dimensions can be associated. For this, links are generated, with link value function (Fig. 9 b). The flat pattern for the foot can be measured starting from the overall dimensions (Fig. 9 c).

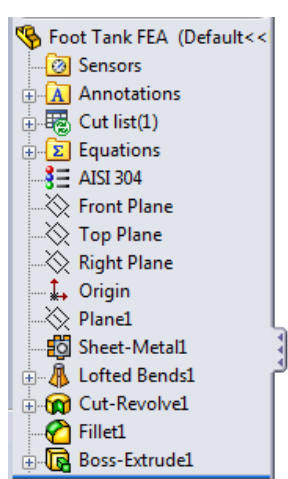

a

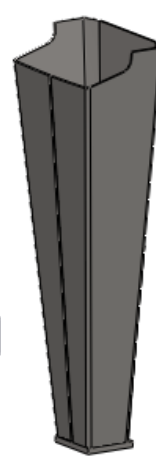

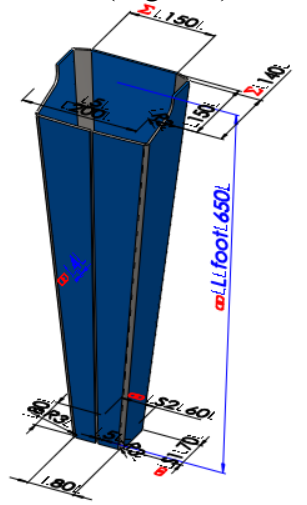

b

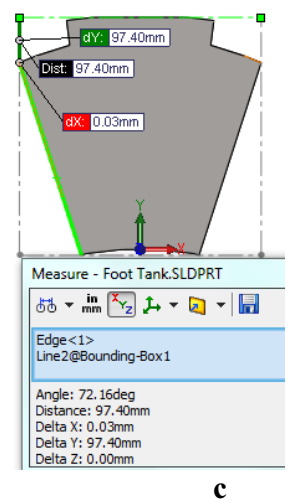

c

Fig. 9. The container foot. 
An important step is to check the feet resistance and possibly there resizing. In this case a finite element analysis is performed on one foot. In order to analyze the response of a component to an applied load, one uses the SolidWorks Simulation finite element analysis (FEA) program. For preparing the analysis model it is very important to define the feet material (alloy steel AISI 304 with Young's Modulus $1.9 \mathrm{e}^{+001}$ and Poisson's Ratio 0.9 N/A) and the external loads.

The containers can have three or four feet. For security reasons at a container with 3 feet one will consider only two. They support the entire container load and in the studied case, the oil with $1.2 \mathrm{~kg} / 1\left(1200 \mathrm{~kg} / \mathrm{m}^{3)}\right.$ density. The total weight, for the studied case, is estimated at $\sim 4955 \mathrm{~kg}$. This corresponds to a calculated pressing force $\sim 48559 \mathrm{~N}$. The force is distributed on the body surface support. It was calculated the stress in the two situations, namely for sheet thickness of $4 \mathrm{~mm}$ (pressure $=32 \mathrm{~N} / \mathrm{mm}^{2}$ ) with a $5 \mathrm{~mm}$ distance in the area of joining sheet foot and similarly for feet with $6 \mathrm{~mm}$ sheet thickness (pressure $=21$ $\mathrm{N} / \mathrm{mm}^{2}$ ) with $1 \mathrm{~mm}$ distance in the area of joining sheet foot.

It was applied Fixed Geometry and Pressure on the two ends of the foot. The result is presented in the Figures 10 and 11.

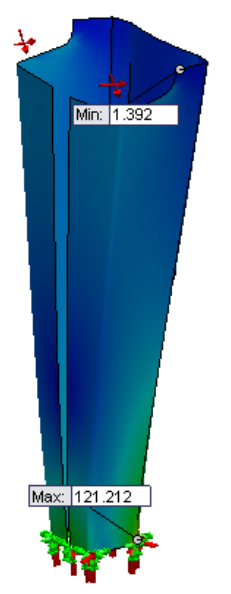

a

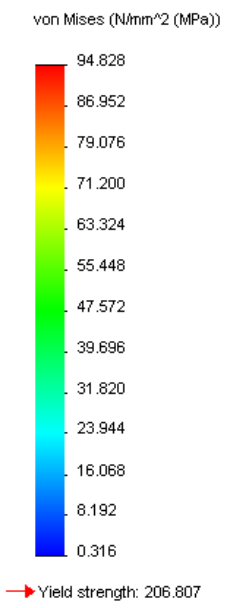

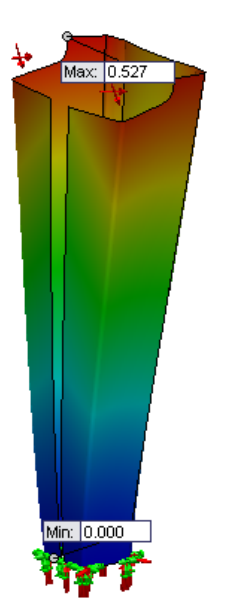

b

Fig. 10. Von Mises (a) and Displacement (b) for $4 \mathrm{~mm}$ sheet thickness.
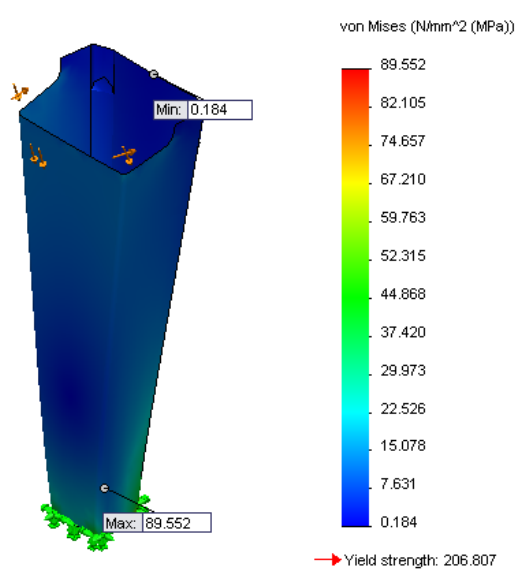

$\mathbf{a}$

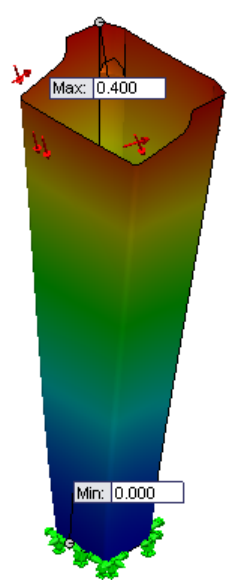

b

Fig. 11. Von Mises (a) and Displacement (b) for $6 \mathrm{~mm}$ sheet thickness. 
Von Mises equivalent stresses, is a scalar quantity obtained from the principal stress values and it is used to measure the state of stress. It is seen that equivalent stress Von Mises is below the maximum allowable flow of the material. Displacement measures the maximum deflection [4]. To avoid all the possible problems, in practical situation, it is preferred the container with $6 \mathrm{~mm}$ sheet thickness.

In order to attach the container pipes, Pipes module is used. SolidWorks provides a library of products required for rapid generation of pipes. From the library named Piping one can attach the necessary components: flanges (Fig. 12 a), valves, elbows, gaskets, equipment. Finally the customized container looks like the Figure below (Fig. 12 b).
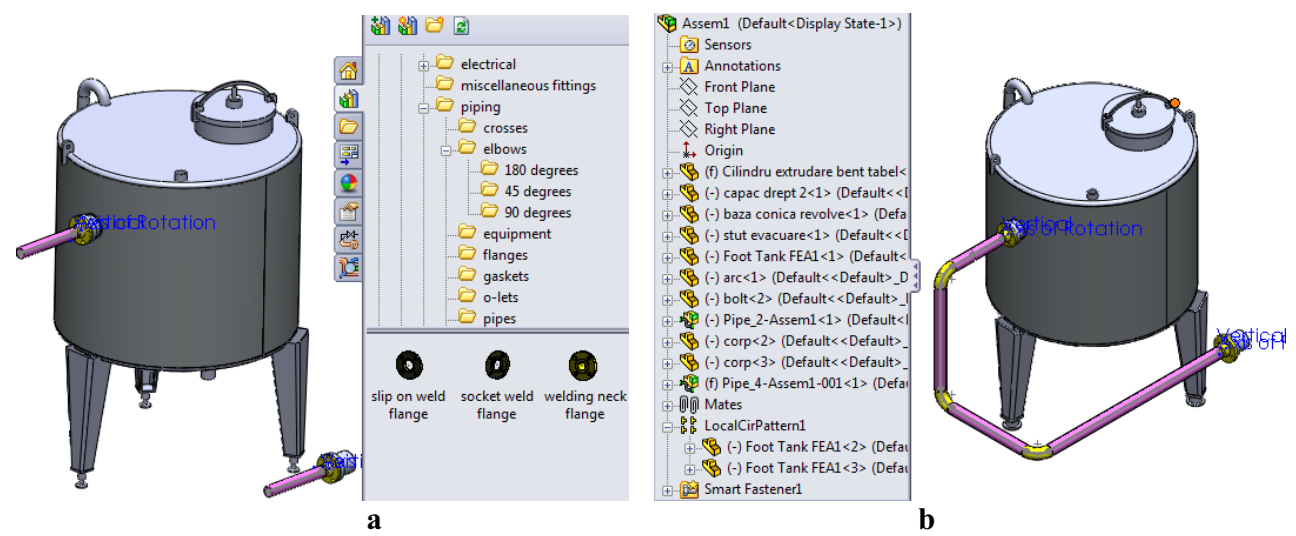

Fig. 12. The container with pipes components.

\section{Other container shapes}

Figure 13 presents an example of a double jacketed (for heating) mixing container, modeled in SolidWorks. This container is equipped with an impeller for mixing. The temperature is controlled with a precision digital thermostat and these containers have a heated stainless steel drain valve with independent temperature control [1].
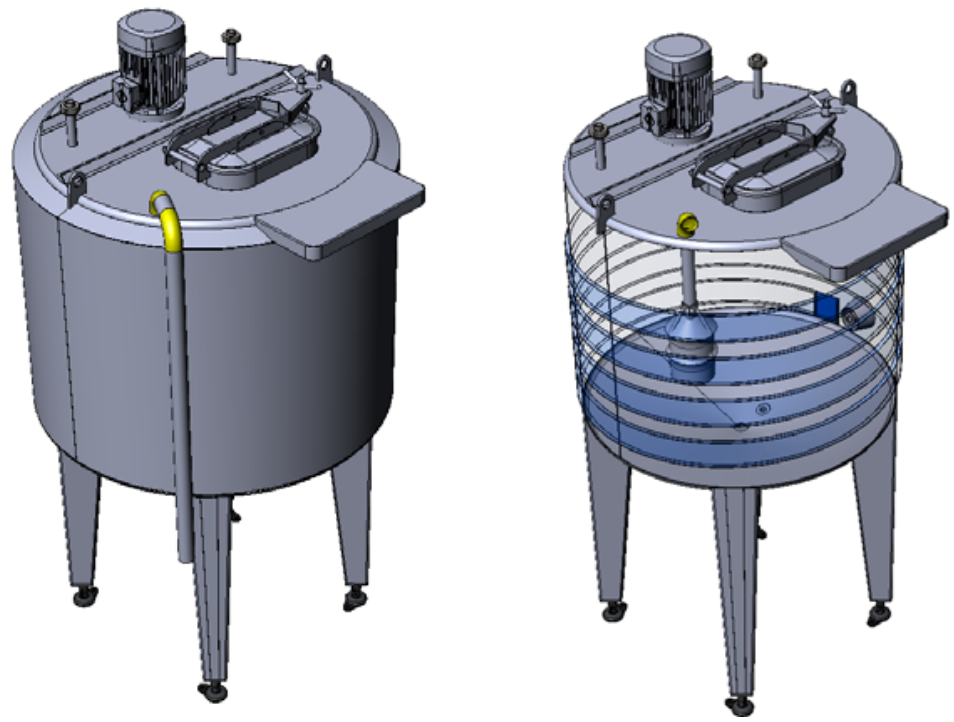

Fig. 13. A double jacketed mixing container. 
Figure 14 a presents an example of a melting container for melting wax for making candles [5]. Melting containers have a hot grid that melts fat blocks and the molten mass is stored inside the container, which has insulation and a hot water jacket [1]. Figure $14 \mathrm{~b}$ presents a melting container modeled in SolidWorks.

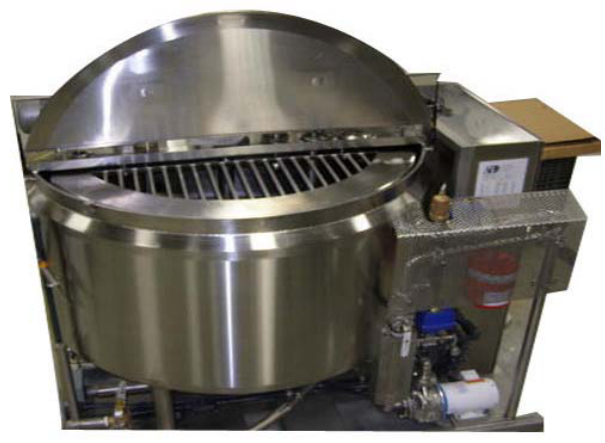

$\mathbf{a}$

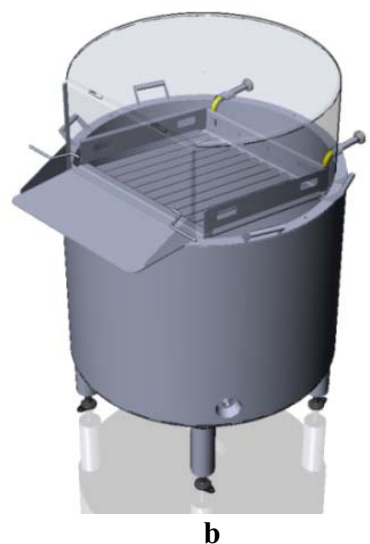

Fig. 14. Melting container.

\section{Conclusions}

The small thickness containers for transport and storage goods, liquids, powder and different raw materials are very used in the food industry. These containers are made of alloy steel sheet in various sizes. The blank material must be properly cut without losses. In this purpose, 3D models have been created in SolidWorks, easily to be flattened and measured.

For the fast modeling of the containers with variable dimensions, the Design Table function has been used. In this activity it was taken into account the size of the blank and the possibility of welding the components.

Certain parts of the container cannot be classically modeled with Sheet Metal functions, namely the container bottom and the cover. The bending area creates this problem. In this case these parts have been modeled before the bending. The length of the sketch, necessary to obtain the desired profile before bending, was calculated. The resulted dimensions were compared with the results from the modeling of the sheets, through Sheet Metal functions.

The resistance of the foot was checked with finite element analysis. In this way it was confirmed the designed foot resistance in the sheet metal area. The containers can be used, after washing, for the storage of other fluids (for example chocolate). Due to this the feet are oversized.

\section{References}

1. http://www.rap-group.ro/raptronic-sisteme-de-proces_en.htm

2. http://help.solidworks.com/2015/English/SolidWorks/sldworks/r_welcome_sw_online help.htm

3. $\bar{E}$., Maican, SolidWorks - 3D modeling for engineers, (Printech Bucureşti, 2006)

4. Gh., L,. Mogan, S., L., Butnariu, Finite element analysis in engineering. Practical applications in CATIA (Transilvania University, 2007)

5. http://www.satyasaifabrications.com/melting-containers.html 